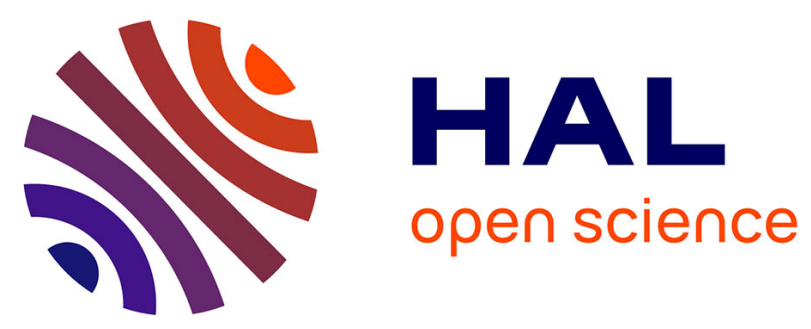

\title{
Can culture confirmation of gonococcal infection be improved in female subjects found to be positive by nucleic acid amplification test (NAAT) in community clinics?
}

Guduru Gopal Rao, Lesley Bacon, Jacqueline Evans, Yemi Dejahang, Ruth Hardwick, Paul Michalczyk, James Wong, Andrés Donaldson

\section{To cite this version:}

Guduru Gopal Rao, Lesley Bacon, Jacqueline Evans, Yemi Dejahang, Ruth Hardwick, et al.. Can culture confirmation of gonococcal infection be improved in female subjects found to be positive by nucleic acid amplification test (NAAT) in community clinics?. Sexually Transmitted Infections, 2009, 85 (7), pp.531. 10.1136/sti.2009.036525 . hal-00552817

\section{HAL Id: hal-00552817 https://hal.science/hal-00552817}

Submitted on 6 Jan 2011

HAL is a multi-disciplinary open access archive for the deposit and dissemination of scientific research documents, whether they are published or not. The documents may come from teaching and research institutions in France or abroad, or from public or private research centers.
L'archive ouverte pluridisciplinaire HAL, est destinée au dépôt et à la diffusion de documents scientifiques de niveau recherche, publiés ou non, émanant des établissements d'enseignement et de recherche français ou étrangers, des laboratoires publics ou privés. 
Can culture confirmation of gonococcal infection be improved in female subjects found to be positive by nucleic acid amplification test (NAAT) in community clinics?

G Gopal Rao ${ }^{1}$, L Bacon ${ }^{2}$, J Evans ${ }^{2}$, Y Dejahang 2 , R Hardwick ${ }^{1}$, P Michalczyk ${ }^{1}$, J Wong $^{1}$, Andrés Donaldson ${ }^{3}$

Microbiology Department, University Hospital Lewisham, London ${ }^{1}$, Department of Community Sexual and Reproductive Health, Lewisham Primary Care Trust, London $^{2}$, Biostatistics Unit, Kings College London Dental Institute ${ }^{3}$

Corresponding Address:

Dr G Gopal Rao

Department of Microbiology, Northwick Park Hospital, Watford Road, Harrow

Middlesex, HA1 3UJ

E-mail: guduru.gopalrao@nwlh.nhs.uk 


\section{ABSTRACT}

Background: Use of nucleic acid amplification tests (NAATs), such as strand displacement assay (SDA, BD ProbeTec ${ }^{\mathrm{TM}}$ C.trachomatis / N.gonorrhoeae Amplified DNA Assay), for the detection of gonococcal infection in the community is controversial because of the possibility of false positive results in low prevalence populations.

Aim: To evaluate if culture confirmation of gonococcal infection can be improved for subjects found to be positive by BD ProbeTec in community clinics.

Methods: Two cervical swabs were collected for culture to confirm NAAT positive results in women age over 16 , a majority of whom were $<25$ years and asymptomatic. One swab (UTP) was urgently transported and processed in the laboratory within two hours whereas the other swab (RTP) was stored at $4^{0} \mathrm{C}$, transported at room temperature and processed 4-72 hours after collection depending on the time and day of collection. Results: 56 subjects with NAAT positive results were recruited into the study. Nine $(16.1 \%)$ subjects who were culture negative were excluded from final analysis due to prior antibiotic treatment (4/9) or the culture having been taken more than one month after the NAAT was positive (4/9) or an incorrect specimen being received (1/9). Overall, 41/47 (87.2\%) NAAT positive subjects were confirmed by culture. 40/47 (85.1\%) UTP swabs and 27/47 (57.4\%) by RTP were positive respectively $(\mathrm{p}<0.05)$.

Conclusion: This study shows that culture confirmation in NAAT positive subjects in a community gonococcus screening programme can be significantly improved by urgent transportation to and processing of specimens in the laboratory. (246 words) Key words: N.gonorrhoeae, Screening, NAAT, BD ProbeTec, Culture 


\section{Introduction}

Nucleic acid amplification tests (NAATs) are highly specific and sensitive tests for the detection of gonococcal infection. ${ }^{1}$ These tests are currently routinely used for screening high risk subjects attending sexually transmitted infection clinics. However there are concerns that these tests may be associated with false positive tests with serious medico-legal consequences in low risk populations. ${ }^{2,3}$ In a recent editorial Ison argues that NAATs for $\mathrm{N}$ gonorrhoeae have not proved as robust as those for $\mathrm{C}$ trachomatis because of the close genetic relatedness to other pathogenic and commensal Neisseria species, The sensitivity and specificity of available NAATs are high but in low prevalence populations, despite the high specificity the positive predictive value may still be unacceptably low. ${ }^{4}$ Therefore Ison and the Centres for Disease Control, Atlanta (CDC) recommend supplementary NAATs should be performed using different target sites to confirm the results of the primary NAAT. ${ }^{4,5}$ Recent studies using supplementary NAATs have shown that primary testing with NAATs are reliable for the diagnosis of gonococcal infection even in community screening programmes and low risk populations. ${ }^{6,7}$

Although NAATs are now acknowledged to be more sensitive than isolation of Neisseria gonorrhoeae by culture, NAATs suffer from a limitation in that they do not provide further information regarding antibiotic sensitivities and molecular epidemiology. ${ }^{4}$ It is being increasingly recognised that in dispersed community clinics, culture of $N$. gonorrhoeae, a fastidious organism, may not always be possible either due to difficulties in obtaining a suitable specimen, on-site culture facilities or absence of adequate transportation facilities that would ensure the survival of the organisms. ${ }^{8}$ In a recent study, we described high prevalence of gonococcal infection using a NAAT (BD 
ProbeTec ${ }^{\mathrm{TM}}$ C.trachomatis/N.gonorrhoea Amplified DNA Assay) in a community based gonococcus screening programme. We were however able to confirm only $67 \%$ of the positive tests by culture. ${ }^{9}$ Similar results were obtained by Moncada et al. ${ }^{6}$ The aim of this study was to evaluate if urgent processing of specimens can improve culture confirmation of specimens obtained from gonococcus NAAT reactive subjects in community clinics.

\section{Methods}

Study Period: April 2007 - December 2008

Location: Four Community Sexual and Reproductive Health (CSRH) clinics in the borough of Lewisham in South London.

Subjects: Female patients over the age of 16 with a positive NAATs test for gonorrhoea form a test who gave informed consent for testing for gonococcal infection. Specimen collection and transportation of specimens for NAAT: Specimens were either taken by the subject or the clinician. Subjects were given detailed instructions for collection of self-taken vulvo-vaginal swabs ( $3 \mathrm{~cm}$ into the vagina). High vaginal or cervical swabs were collected if vaginal examination was indicated. The swabs used for NAAT were provided by the manufacturers (BD ProbeTec ${ }^{\mathrm{TM}}$

C.trachomatis/N.gonorrhoea Amplified DNA Assay Endocervical and Urethral Collection and Dry transport kits, Becton Dickinson, UK).

Laboratory Tests: Tests were performed in the microbiology department at University Hospital Lewisham. The NAAT used was based on strand displacement amplification assay (SDA, BD ProbeTec ${ }^{\mathrm{TM}}$, Becton Dickenson, UK). All positive tests were repeated in the laboratory to confirm the results and exclude contamination during testing. A result was reported positive only if both tests were positive.

Specimen collection and transportation of specimens for culture of $N$. gonorrhoeae: 
Subjects with positive NAAT for N.gonorrhoeae were recalled to the clinics as soon as possible. Two cervical swabs and a urethral swab from each patient (Transport swab, Amies medium with charcoal, Mediwire, UK) were taken by the clinician prior to commencement of treatment for culture and antibiotic sensitivity tests. Participants over age 16 who agreed to take part in the study gave specific informed written consent for the extra cervical swab to be taken.

The swab sent for routine transportation and processing (RTP) was stored at $4{ }^{\circ} \mathrm{C}$ in the clinic and transported to the laboratory at room temperature on the following day by a routine laboratory courier system which collected the swabs from the clinics between 1100 - 1300 hours, Monday - Friday. These swabs were delivered and processed in laboratory (for culture and antimicrobial sensitivity) between 1400-1700 hours on the same day. Swabs collected from evening clinics on Friday and from Saturday morning clinics were stored at at $4^{0} \mathrm{C}$ and processed in the afternoon on Monday. These procedures reflected the routine practice of the community clinics.

The swab sent for urgent transportation at room temperature and processing (UTP) was sent to and processed in the laboratory within two hours of collection of the specimen. In the laboratory, the swabs were cultured on selective Gonococcus Medium (Lysed GC Selective Agar Ref. PB0962A, Oxoid, UK) for N.gonorrhoeae and incubated in $10 \% \mathrm{CO}_{2}$ at $37 \mathrm{C}$ for 48 hours. Oxidase positive colonies were confirmed as N.gonorrhoeae by Gram stain, biochemical (Neiseria $\mathrm{PET}^{\mathrm{TM}}$, Bioconnections, UK) and immunological tests (Gonogen $\mathrm{II}^{\mathrm{TM}}$, Bioconnections, UK).

Statistical Methods: The McNemar test was used to compare the probabilities of a misclassification of result between the RTP and UTP methods.

Ethical approval: Ethical approval was obtained form the Lewisham Ethics Committee. 


\section{Results:}

A total of 56 NAAT reactive subjects were recruited into the study.

Nine (16.1\%) subjects who were culture negative were excluded from final analysis.

These included mainly treatment with antibiotics prior to collection of specimens for culture for either having chlamydia infection themselves or being a contact of a subject with chlamydia infection (4/9) or the culture was taken greater than one month after the NAAT was positive (4/9). In one patient (1/9), both cervical swabs were mistakenly sent by routine transport and processed routinely but the urethral swab which was sent urgently was positive by culture. There were no positive tests that failed to be confirmed by repeat testing.

$47 / 56(83.9 \%)$ subjects were included in the final analysis. The clinical notes of two patients could not be traced. Most of the subjects (39/45, 87\%) were under 25 years of age. A majority of the subjects were asymptomatic $(36 / 45,80 \%)$. Those who had symptoms $(9 / 45,20 \%)$ included discharge (3), per vaginal bleeding with Implanon (3), dysuria (1), abdominal pain (1) and test of cure for a previous gonococcal infection (1). 41/47 (87.2\%) NAAT reactive subjects were positive by culture. Whereas 40/47 ( $85.1 \%$ ) were positive by the UTP method, only $27 / 47$ (57.4\%) were positive by the RTP method. In one subject, culture by RTP method was positive but negative by UTP method. McNemar's test with the continuity correction yields a Chi squared(1df)=9.6; $\mathrm{P}=0.002$.

Of the six subjects $(6 / 47,12.8 \%)$ in whom RTP and UTP cultures were negative, four subjects had no antibiotic treatment prior to culture, one subject was treated with trimethoprim for urinary tract infection. (unlikely to affect culture as N.gonorrhoeae are resistant to trimethoprim and in one subject the urethral swab was positive by culture. 


\section{Discussion:}

Use of nucleic acid amplification tests (NAATs) such as BD ProbeTec ${ }^{\mathrm{TM}}$ for the detection of gonococcal infection in low prevalence population may lead to false positive results. ${ }^{2}$ To illustrate this, Ison ponts out that even NAATs with relatively high specificity (99\%) may result in relatively low positive predictive value $(50 \%)$ of the test in populations where the prevalence is only $1 \% .{ }^{4}$ Culture confirmation of the NAAT reactive results in dispersed clinics in the community is challenging because of rapid loss of viability of gonococcus during any delay in transportation and processing of the laboratories. ${ }^{8}$ In a recent paper describing our experience of screening in the community using BD ProbeTec ${ }^{\mathrm{TM}}$, we reported an unexpectedly high prevalence (3.8\%) of gonococcal infection in the Lewisham community. ${ }^{9}$ At the time of reporting the results, we were concerned whether some of the NAAT reactive positive results were 'false positive' as only $67 \%$ of these results could be confirmed by culture or whether the cultures were false negative due to loss of viability of the organisms during transportation and processing. In a recent paper we showed that over $97 \%$ of BD ProbeTec ${ }^{\mathrm{TM}}$ reactive tests could be confirmed by supplementary NAATs.

However confirmation by supplementary NAATs does not necessarily address the issue of false negative culture tests in subjects with reactive NAAT tests. We believe that culture not only serves to confirm the results of NAAT but enables determination of antibiotic sensitivity and study of the molecular epidemiology of the infection.

A majority (>80\%) of the subjects included in this study were under 25 years and asymptomatic and screened as a part of the Lewisham Chlamydia and Gonococcus Screening Programme. In order to address the issue of false negative culture tests, we have attempted to evaluate if culture confirmation of NAAT can be improved in community screening programmes by comparing urgent transportation and processing (UTP) with 
routine urgent transportation and processing (RTP). Overall, $41 / 47$ (87.2\%) NAAT positive subjects were confirmed by culture of cervical swabs.

UTP can significantly increase culture confirmation (40/47, 85.1\%) compared to RTP (27/47, 57.4\%). We are unable to explain why in one subject the RTP swab was positive for culture and the UTP swab negative. It is conceivable that it may be related to the amount of the specimen material on the swabs. The detection of one subject where the urethral swab was culture positive and the cervical swabs were negative, confirms that in at least in some subjects, collection of urethral swabs may further improve the culture confirmation of NAAT where the cervical swab may be negative. ${ }^{10}$ Including the positive urethral swab, the overall culture confirmation rate was $89.4 \%$ (42/47). Currently community clinics in Lewisham do not have the resources to directly inoculate swabs on culture media and incubate the inoculated media at $37^{\circ} \mathrm{C}$ in $10 \% \mathrm{CO}_{2}$ prior to transportation to the microbiology laboratory.

Our study also shows that culture confirmation is feasible in a majority of the subjects even in dispersed clinics if the swabs are transported and processed urgently in the laboratory. We acknowledge that UTP may not be convenient or possible for many dispersed clinics due to the logistics or insufficient resources. Under such circumstances we recommend that periodical 'culture' surveys are undertaken in the community when the specimens are transported and processed urgently. This will ensure that there is surveillance of antibiotic resistance in the community strains, which is crucial for determining the empirical treatment of choice for gonococcal infections in the community. In conclusion, this study shows that culture confirmation of NAAT reactive subjects in community clinics can be significantly improved by urgent transportation and processing of specimens in the laboratory.

(1722 words) 
Acknowledgement: We thank all the members of the Lewisham Chlamydia and Gonococcus Screening Group and staff of the Microbiology Laboratory at University Hospital Lewisham for their support.

Funding: This study was supported by a grant from the Lambeth, Southwark and Lewisham Research Scheme. 


\section{References}

1. Van Der PB, Ferrero DV, Buck-Barrington L, et al. Multicenter evaluation of the BDProbeTec ET System for detection of Chlamydia trachomatis and Neisseria gonorrhoeae in urine specimens, female endocervical swabs, and male urethral swabs. J Clin Microbiol 2001;39:1008-16.

2. Akduman D, Ehret JM, Messina K, et al. Evaluation of a strand displacement amplification assay (BD ProbeTec-SDA) for detection of Neisseria gonorrhoeae in urine specimens. J Clin Microbiol 2002;40:281

3. Barlow D. Commentary: prevalence of Neisseria gonorrhoeae infection in young subjects attending community clinics in South London. Sex Transm Infect 2007;84:121.

4. Ison C. GC NAATs: is the time right? Sex Transm Infect 2006;82:515.

5. Centers for Disease Control and Prevention. 2002. Screening tests to detect Chlamydia trachomatis and Neisseria gonorrhoeae infections-2002. MMWR Morb. Mortal. Wkly. Rep. 51:1-38

6. Moncada J, Donegan E, Schachter J . Evaluation of CDC-recommended approaches for confirmatory testing of positive Neisseria gonorrhoeae nucleic acid amplification test results. J Clin Microbiol. 2008 May;46(5):1614-9

7. R Hardwick, G Gopal Rao, H Mallinson. Confirmation of BD ProbeTec Neisseria gonorrhoea reactive samples by Gen-Probe APTIMA assays and culture. Sex Transm Infect. 2009;85;24-26

8. Iwen PC, Walker RA, Warren KL, et al. Effect of off-site transportation on detection of Neisseria gonorrhoeae in endocervical specimens. Arch Pathol Lab Med 1996;120:1019-22. 
9. Gopal Rao G, Bacon L, Evans J, et al. Prevalence of Neisseria gonorrhoeae infection in young subjects attending community clinics in South London. Sex Transm Infect 2007;84:117-21.

10. Lavelle SJ, Jones KE, Mallinson H, Webb MC. Finding, confirming, and managing gonorrhoea in a population screened for chlamydia using the GenProbe Aptima Combo2 assay. Sex Transm Infect 2006;82:221-24. 


\section{Key Messages}

1. Culture Confirmation of Nucleic Acid Amplification Tests (NAATS) can be significantly improved even in dispersed community clinics by urgent transportation and processing of specimens.

2. Generally, failure to culture N.gonorrhoeae from specimens in NAAT positive subjects in the community is likely to be a result of loss of viability of the organisms rather than the NAATs being 'false positive'.

3. Culture confirmation of NAAT can provide useful information regarding antibiotic resistance and molecular epidemiology N.gonorrhoeae which will be important in the management of gonococcal infections in the community. 


\section{Contributions of the authors:}

G.Gopal Rao: Concieved the project, contributed to the methodology, analysis and manuscript and is the guarantor of the study.

L. Bacon: Concieved the project, contributed to the methodology, specimen and data collection, analysis and manuscript.

Y. Dejahang: Contributed to methodology, specimen and data collection.

J. Evans: Contributed to methodology, specimen and data collection.

R. Hardwick: Contributed to methodology, laboratory examination, and data analysis J. Wong: Contributed to methodology and laboratory examination

P. Michalczyk: Contributed to methodology, laboratory examination and data collection and analysis.

Andres Donaldson: Performed the statistical analysis and contributed to the manuscript. 
"The Corresponding Author has the right to grant on behalf of all authors and does grant on behalf of all authors, an exclusive licence (or non exclusive for government employees) on a worldwide basis to the BMJ Publishing Group Ltd to permit this article (if accepted) to be published in STI and any other BMJPGL products and sub-licences such use and exploit all subsidiary rights, as set out in our licence http://sti.bmjjournals.com/ifora/licence.pdf)". 
